\title{
What a Johnson or Corbyn government might mean for the NHS
}

In this Observations article (BMJ 2019;367:16557; doi:10.1136/ bmj.16557) the 28 June 2016 parliamentary Labour Party vote of no confidence was given as 72-40 instead of 172-40. This has now been corrected. 